$\mathrm{E}$ EVALUAR
2017, Vol. 17, No 2.

ISSN $1667-4545$

Recuperado de https://revistas.unc.edu.ar/index.php/revaluar

Laboratorio de Evaluación Psicológica y Educativa

Facultad de Psicología - Universidad Nacional de Córdoba

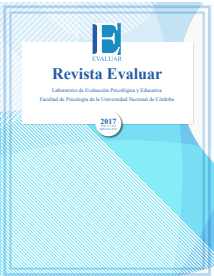

\title{
Propiedades Psicométricas del Cuestionario de Adaptación para Adolescentes Bell en Población Bogotana
}

\author{
Psychometric Properties of the Bell Adolescent Adaptation \\ Questionnaire in Bogota Population
}

\author{
Rocío del Pilar Gómez-Ramírez * ${ }^{1}$, Guadalupe de la Iglesia ${ }^{2}$ \\ 1 - Universidad Nacional de Colombia, Colombia. \\ 2 - Universidad de Buenos Aires, Argentina.
}

Introducción

Método

Análisis de datos

Resultados

Referencias

Recibido: 18/07/2017 Revisado: 29/07/2017 Aceptado: 12/08/2017

\section{Resumen}

El presente trabajo tuvo como objetivo establecer las propiedades psicométricas del Cuestionario de Adaptación para Adolescentes de Bell (Bell Adjustment Inventory; 1934), cuyo fin es operacionalizar el grado de adaptación en áreas relevantes del desarrollo adolescente. La muestra estuvo compuesta por 569 adolescentes entre 12 y 18 años residentes en la ciudad de Bogotá, Colombia. Se obtuvieron evidencias de validez de constructo por medio de un análisis factorial, cuyos resultados sugirieron mantener la estructura de cuatro dimensiones de adaptación: familiar, de salud, social y emocional. Se evaluó la consistencia interna de cada dimensión calculando los coeficientes alfa de Cronbach. Además, se estudiaron diferencias según sexo y edad. Los resultados obtenidos en cuanto a validez de constructo $\mathrm{y}$ consistencia interna son favorables e indican un buen funcionamiento psicométrico del instrumento.

Palabras clave: adaptación, adolescentes, propiedades psicométricas, pruebas psicológicas

\begin{abstract}
The aim of this study was to establish the psychometric properties of the Bell Adjustment Inventory for adolescents (Bell, 1934), an instrument that aims to operationalize the degree of adaptation in adolescents in different relevant areas of their development. The study was conducted on a sample of 569 adolescents between 12 and 18 years old residing in the city of Bogota, Colombia. The results showed evidence of construct validity through a factor analysis by keeping its structure of four adjustment dimensions that correspond with family, health, social and emotional. The internal consistency of each dimension was evaluated by calculating the Cronbach alphas. Also, differences depending on sex and age were studied. The results indicated a proper psychometric functioning of the instrument and evidences of construct validity were obtained as well as evidences of good internal consistency.
\end{abstract}

Keywords: adjustment, adolescent, psychometric properties, psychological test

*Correspondencia a: Rocío del Pilar Gómez Ramírez, Calle 39 BIS \#29-90 Bogotá, Colombia. Te.: +573003066063. rdgomezr@unal.edu.co Cómo citar este artículo: Gómez-Ramírez, R. del P., de la Iglesia, G. (2017). Propiedades psicométricas del Cuestionario de Adaptación para Adolescentes Bell en población bogotana. Revista Evaluar, 17(2), 47-66. Recuperado de https://revistas.unc.edu.ar/index.php/revaluar Nota: El presente artículo es derivado de la investigación "Propiedades psicométricas del Cuestionario de Adaptación para Adolescentes de Bell en población bogotana" presentada como tesis de grado de maestría. 


\section{Introducción}

La adaptación es reconocida como función comportamental universal y atributo interno de la conducta y está relacionada con el bienestar puesto que influye en la estabilidad emocional y personal (Jiménez \& López-Zafra, 2011; LabajosAlonso, 1996; Rodríguez, Jiménez, Fernández, \& Godoy, 1999). Diferentes autores la definen como el proceso en el que un organismo vivo mantiene el equilibrio entre sus necesidades y las circunstancias que influyen en cómo estos logran satisfacerlas (Kulshrestha, 1979; Petersen \& Crockett, 1985). En el proceso adaptativo el organismo se ajusta a un ambiente que se encarga de moldearlo, otorgándole rasgos que benefician su exploración.

A la vez, se ha descrito la adaptación como la respuesta a cambios o conflictos generados por factores internos (necesidades básicas como alimentación, descanso, estado de salud, entre otros) o externos (procesos de duelo, afrontamiento hacia distintos estímulos, ajuste social, entre otros) a partir del conocimiento de las necesidades propias (Bharvad, 2015; Cardenal-Hernáez \& FierroBardaji, 2001; Del Bosque-Fuentes \& AragónBorja, 2008; Holahan, Moos, \& Schaefer, 1996; Macías, 2000).

De acuerdo con lo anterior, la adaptación está constituida por un doble proceso: la tendencia al ajuste de las personas a los propios deseos, gustos, preferencias y necesidades; y el ajuste de las propias conductas a las circunstancias de un entorno con normas y pautas en las relaciones interpersonales en el que se hallan insertos. En el doble proceso, el individuo no está aislado ni contrapuesto al mundo, sino por el contrario, siempre relacionado con él (Alcalay, Milicic, \& Torretti, 2005; Fierro \& Cardenal, 1996). Los mecanismos de ajuste que median la adaptación parten de conductas y cogniciones propias del sujeto, proponiendo un acuerdo entre las características personales y las demandas del medio al que pertenece (García-Pérez \& Magaz-Lagos, 2001; Gómez-Bustamante \& Cogollo, 2010; MartínezFerrer, Amador-Muñoz, Moreno-Ruiz, \& MusituOchoa, 2011; Orte-Socías \& March-Cerdá, 1996).

De esta forma se define la adaptación como un constructo relacional por ser un proceso activo e interactivo de ajuste a las necesidades y demandas propias y del entorno para hacer a este último habitable. La adaptación constituye, por ende, un hábito comportamental y un estado emocional (García-Pérez \& Magaz-Lagos, 2001; Fierro, 1990, 1997; Fierro \& Cardenal, 1996; Kulshrestha, 1979; Ochoa, 2012; Siverio-Eusebio \& García-Hernández, 2007). Es así como la adecuada adaptación ocurre tanto por el reconocimiento de las emociones y habilidades personales como por el mantenimiento de relaciones personales, familiares y extrafamiliares adecuadas, lo cual permite explorar el mundo social y personal, así como conocer y apropiar formas de comportamiento relacionadas con la idiosincrasia de un contexto.

Generalmente una adecuada adaptación se relaciona con altos niveles de autoestima, autoimagen y autoconcepto; adecuado desempeño académico y profesional y satisfacción vital (Kostanski \& Gullone, 1998; Musitu \& García 2001). De esta manera, las personas que logran una adecuada adaptación se caracterizarían por poseer altos niveles de autonomía e independencia con tendencias a la dinámica y vinculación relacional, satisfacción con su cotidianeidad y adecuada resolución de dificultades (Fierro, 1997; Fierro \& Cardenal, 1996; Kulshrestha, 1979; Ochoa, 2012; Siverio-Eusebio \& GarcíaHernández, 2007). 
La adaptación en la adolescencia

La adaptación se da en todas las etapas de desarrollo y determina la calidad de vida a nivel biológico, emocional, psicológico y social. Frente a esto se ha descrito que el logro de la adaptación en la adolescencia es fundamental (CarvajalCorzo, 1993; Gómez-Bustamante \& Cogollo, 2010; Labajos-Alonso, 1996). Se entiende por adolescencia una etapa del ciclo vital del desarrollo entre la infancia y la adultez donde se destacan la apropiación de una identidad y la vinculación afectiva, que resultan en la inserción en un grupo social de pertenencia (Carvajal-Corzo, 1993). Es evidente que la adaptación es de especial importancia en esta etapa en la que se da una serie de inmensos cambios inicialmente biológicos y posteriormente psicológicos.

El estudio de la adaptación adolescente se enfoca en la estabilidad personal, la autopercepción emocional y del estado físico y las interacciones sociales (Coleman \& Hendry, 1999; González, De los Ríos, \& Viveros, 2015; Lynch, Norem, \& Gergen, 1981). En esta etapa, la adaptación se consolida a través de variables mediadoras, a saber: biológicas, que representan el inicio de la pubertad afín a los cambios corporales, el temperamento y estados de salud en general y las cuales se desprenden del desarrollo orgánico y físico-psicosexual; psicológicas, relacionadas con el estado emocional, inteligencia, autopercepción, desarrollo del ego, desarrollo moral y desarrollo cognoscitivo; y sociales, en las que se denotan las nuevas configuraciones en la relaciones de pares y la entrada al mundo social y cultural (Alcalay et al., 2005; Coleman \& Hendry, 1999; Fierro, 1990).

La evidencia empírica a la fecha sugiere que la edad es un factor relevante asociado a la adaptación. Esto es debido a que la etapa adolescente se divide en tres subetapas según rangos de edad.
Cada una de estas cumple un papel importante en el desarrollo, ya que unifica características fisiológicas, reacciones y conductas comunes presentadas en los adolescentes de acuerdo con el proceso de desarrollo (Carvajal-Corzo, 1993; Ros-Rahola et al., 2006). En la Tabla 1 se encuentran condensadas las características de cada una de las etapas de la adolescencia según Carvajal-Corzo (1993).

Asimismo, el sexo es otro factor relevante en la adaptación adolescente. En estudios referidos a las diferencias según sexo, por ejemplo, se ha descrito que las mujeres adolescentes muestran un mayor grado de inadaptación que los varones en un ámbito educativo, ya que estas se perciben como víctimas frente a la hostilidad de pares, mientras que los hombres tienden a la formación de grupos, observándose a sí mismos como ajustados al entorno (Funes, Lupiáñez, \& Humphreys, 2010). Estos hallazgos coinciden con los descritos por Aragón-Borja y Bosques (2012), según los cuales los hombres adolescentes refieren mayores índices de adaptación debido a la poca importancia que le dan a los cambios físicos y, por lo mismo, a la autoimagen percibida en la inmersión en grupos, en los que la comunicación con pares trabaja como factor relevante para los procesos de adaptación.

Sin embargo, otros estudios señalan mayores índices de adaptación en las mujeres adolescentes que en los hombres en lo que refiere a la necesidad de ajustarse a los cambios que implica esta etapa, especialmente aquellos vinculados con los cambios corporales. Para estos autores el hecho de que el cuerpo femenino se desarrolle a mayor velocidad y este desarrollo sea más evidente que en los hombres propende al afán por ajustarse a las presiones, a los patrones sociales y al grupo de pares. Asimismo, las mujeres parecen recibir la etapa adolescente con reflexiones sobre lo que deviene de la misma, al contrario que los hombres (Bharvad, 2015; Del Bosque-Fuentes \& Aragón- 
Tabla 1

Etapas del período adolescente (Carvajal-Corzo, 1993).

Caracterizada por los primeros cambios físicos, a saber: a) el aumento de la estatura, b) el aumento del peso corporal y de fuerza muscular, c) el cambio de voz y d) la aparición de los caracteres sexuales secundarios.

Se destaca la preocupación de los adolescentes por los cambios corporales y de autoconcepto e imagen, por lo que surgen frecuentemente las comparaciones frente a pares del mismo sexo. A la par sucede el incremento de integración y contacto con

Adolescencia Primaria (12 - 13 años) personas del mismo sexo, se construyen los primeros índices de búsqueda de identidad a través de la pertenencia de grupos de amigos y lazos fraternales, así como las primeras sensaciones eróticas y la necesidad de intimidad.

Se dan las primeras manifestaciones de ambivalencia emocional, especialmente la necesidad de independencia y nostalgia por la dependencia llevada hasta el momento con los padres.

En cuanto al nivel cognitivo, en esta etapa se produce la evolución del pensamiento abstracto, la idealización de las vocaciones; se da menos control de los impulsos y mayor entrada al mundo de las fantasías.

Se caracteriza por la remodelación morfológica y la conformación de la identidad sexual. Aumenta la atracción por el sexo opuesto, por lo que surgen actividades en pro del cuidado del cuerpo y la figura.

En el área cognitiva se inicia el pensamiento abstracto y reaparecen manifestaciones

Adolescencia Nuclear (14 a 15 años)

\section{egocéntricas.}

Se incrementan los conflictos con padres y autoridades, ya que se anhela la trasgresión de las normas, se da mayor fuerza a las opiniones propias y aumenta el sentido de independencia y la rebeldía.

Se busca la independencia, el adolescente se percibe a sí mismo como poderoso y fuerte, características que llevan a comportamientos agresivos y en ocasiones peligrosos, como el consumo de drogas, el fumar, la ingesta de alcohol, entre otros.

Esta marca el final del período adolescente. En ambos sexos se alcanzan las características del adulto en cuanto a funciones plenas para la sexualidad y reproducción. Se acepta la propia imagen y se establecen preferencias de amigos, música, forma de vestir, entre otras.

Adolescencia tardía

(16 a 18 años)

En el área social se vuelve a apreciar el valor y consejo de los padres y se da la creciente integración en el medio social. Se establecen las relaciones íntimas, como la formación de parejas y amistades cercanas, relaciones que se caracterizan especialmente por el compartimiento de experiencias.

Se presenta la maduración cognitiva y la convivencia emocional en donde se logra el control de impulsos emocionales, la capacidad para comprometerse y establecer límites y la concreción de valores y objetos personales.

Borja, 2008; Alonso-Fernández, 2005).

En cuanto la edad como factor adaptativo, Cardenal-Hernáez y Fierro-Bardaji (2001) no encontraron diferencias significativas entre la adolescencia temprana, la nuclear y la tardía en cuanto a la adaptación evaluada entre hombres y mujeres. Sin embargo, describen que los adolescentes entre 16 y 18 años presentan mayores ín- dices de ajuste orientados a la protección de los demás, la reflexión sobre proyectos de vida y el conformismo. Se afirma que esto ocurre así debido a que en la adolescencia tardía los jóvenes se acercan al final del período, entrando al proceso de ajuste adulto. Asimismo, Aragón-Borja y Bosques (2012) hallaron que a mayor edad, mayores índices de adaptación adolescente, es decir, los 
jóvenes establecidos en la fase de adolescencia tardía tienden a estar más adaptados. Por su parte, los jóvenes en el período de adolescencia nuclear presentan mayores índices de inadaptación por estar atravesando mayores cambios durante esta fase y tienden a la introversión y al poco reconocimiento de sí mismos.

\section{Operacionalizaciones de la adaptación}

A pesar de la importancia de la adaptación en la adolescencia, son escasos los instrumentos construidos para su medición en esta etapa. En la actualidad las escalas desarrolladas no se enfocan exclusivamente en la medición de la adaptación sino que valoran subaspectos relacionados o constructos similares entre los que están: la evaluación de la autoestima, para la cual se han desarrollado el Cuestionario de Autoestima Global de Rosenberg (1966) y el Inventario de Depresión Infantil (CDI) de Kovacs (2004), la evaluación de autoeficacia, en la que se destaca la Escala de Autoeficacia General de Baessler y Schwarzer (1996), la evaluación de la satisfacción con la vida, para la que se encuentran la Escala de Satisfacción con la Vida de Diener, Emmons, Larsen y Griffin (1985), su estandarización española realizada por Atienza-González, Moreno-Sigüenza y Balaguer-Solá (2000) y la Escala Multidimensional de Satisfacción con la Vida de Huebner (1991); la evaluación del clima escolar con la Escala de Clima Escolar (Moos, 1974), la adaptación española de Fernández-Ballesteros y Sierra (1984); y la evaluación de las habilidades sociales, para la cual existen la Escala de Actitudes y Estrategias Cognitivas Sociales (AECS) de Moraleda, González-Galán y García-Gallo (1998) y la Escala Multidimensional de Expresión Social (EMES) de Caballo y Ortega (1989), adaptada por Gutiérrez (2000). Estos instrumentos, a pesar de ser relevantes por estu- diar aspectos de la conducta y eficacia adolescente, no brindan una medida general de adaptación. Por su parte, el Cuestionario de Adaptación para Adolescentes de Bell (CAAB; Bell, 1934) es un instrumento específicamente diseñado para evaluar la adaptación adolescente.

\section{Cuestionario de Adaptación}

para Adolescentes de Bell

El Cuestionario de Adaptación para Adolescentes de Bell (CAAB; Bell, 1934) evalúa el estado general de adaptación adolescente de acuerdo con el modelo de adaptación propuesto por su autor según el cual son cuatro las dimensiones representativas del proceso de adaptación adolescente: familiar, social, de la salud y emocional. Estas dimensiones no son excluyentes entre sí, por el contrario interactúan, propiciando el referente de estabilidad emocional y el adecuado desenvolvimiento en un contexto determinado.

La adaptación familiar indica el grado de satisfacción del adolescente con los padres, principalmente, y su familia en general (Bell, 1934). La adaptación social se refiere al establecimiento y mantenimiento de relaciones personales afectivas adecuadas y el dominio de tareas relacionadas con la edad. Un buen desempeño social proporciona el sentido de pertenencia al contexto cultural, lo cual hace que el adolescente perciba satisfacción general dentro de las esferas sociales que lo rodean (Bell, 1934; Cerdá, 1987; Jiménez \& López-Zafra 2011; Valverde, 1988). La adaptación de la salud es definida como un estado completo de adecuación física, lo que implica no sólo la ausencia de afección o enfermedad, sino la total cobertura de las necesidades fundamentales de manera sanitaria y nutricional. Este tipo de adaptación refiere a la adecuada condición de salud física, orgánica y corporal (Palmero \& Fernández, 1998). Final- 
mente, la adaptación emocional se relaciona con el grado de reconocimiento por parte del adolescente tanto de sus propios sentimientos como de los de los demás para manejarlos y controlarlos adecuadamente (Bell, 1934; Lynch et al., 1981; Oliva-Delgado \& Parra-Jiménez, 2004; Valverde, 1988).

Los primeros trabajos psicométricos del cuestionario fueron realizados por Bell (1934), quien seleccionó cada una de las dimensiones del cuestionario partiendo del grado en que diferenciaban entre el $15 \%$ de individuos superior y el $15 \%$ inferior en una distribución de puntuación obtenida en la aplicación piloto del cuestionario de adaptación. Únicamente los ítems que diferenciaban claramente los grupos extremos se incluyeron en la construcción del cuestionario para la versión en inglés.

Para la validación, el cuestionario fue administrado a un grupo de estudiantes que además fueron calificados en los correspondientes sectores de adaptación por directores y psicólogos escolares. La escala de adaptación social se validó mediante la correlación Pearson con la escala adaptación social del Cuestionario de Ascendencia-Sumisión de Allport y Allport (1939) y B4-D del cuestionario de personalidad de Bernreuter (B4-D; Bernreuter, 1935), y la escala adaptación emocional y la puntuación total se validaron buscando también la correlación con el Personality Schedule de Thurstone (Thurstone, 1930). Los coeficientes de correlación obtenidos fueron: Cuestionario de Ascendencia-Sumisión y adaptación social (hombres) $=.72$; Cuestionario de Ascendencia-Sumisión y adaptación social (mujeres $)=.81$; Personality Schedulede Thurstone $\mathrm{y}$ adaptación emocional $=.93$, Personality Schedulede Thurstone y puntuación total $=.94$; Cuestionario de personalidad de Bernreuter y adaptación social $=.90$.

Para corroborar la confiabilidad se calcula- ron coeficientes $\rho$ para cada una de las dimensiones del cuestionario y del puntaje global a través de la fórmula de Spearman-Brown. La puntuación fue significativa: adaptación familiar, $\rho=$ .89 , adaptación de la salud, $\rho=.80$; adaptación social, $\rho=.89$; adaptación emocional, $\rho=.85 \mathrm{y}$ puntuación total, $\rho=.93$

Finalmente Bell (1934) para la validación del instrumento, realizó intercorrelaciones para las cuatro escalas del cuestionario obteniendo: Familia y Salud $=.43$; Familia y Social $=.04 ; \mathrm{Fa}-$ milia y Emocional $=.38$; Salud y Social $=.24$; Salud y Emocional $=.53 \mathrm{y}$, Social y Emocional $=.47$

En la versión española adaptada por Cerdá (1987) se modificó el texto de 14 ítems en concordancia con el contexto español, para mantener igual número de ítems del cuestionario original. Los coeficientes de confiabilidad son de $\alpha=.89$ para la escala de adaptación familiar, $\alpha=.80$ para la escala de adaptación en salud; $\alpha=.89$ para la escala de adaptación social y $\alpha=.84$ para la escala de adaptación emocional. En cuanto a la validez de contenido y de constructo, se analizó cada uno de los ítems en cuanto a conceptos de acuerdo con el contenido de cada una de las dimensiones. Se utilizó la matriz de correlación inter-subescala y se detectó una correlación negativa alta entre la adaptación y no adaptación, es decir, adecuados índices psicométricos para la versión española.

En un estudio con una versión mexicana del instrumento se trabajó con una muestra de 562 estudiantes de la Ciudad de México. Para esta adaptación se utilizaron las mismas evaluaciones que para la adaptación española, calculando el índice de confiabilidad para cada una de las escalas $(\alpha$ $=.80$, para el área familiar, $\alpha=.75$ para el área de salud, $\alpha=.71$ para el área social, $\alpha=.85$ para el área emocional y $\alpha=.92$ para el total del cuestionario). En este trabajo, la escala resultante mantuvo la misma estructura de la adaptación española (Del Bosque-Fuentes \& Aragón-Borja, 2008). 
En países como Perú, Ecuador y Chile se ha utilizado la versión española del CAAB para diferentes estudios, pero no se llegó a adaptar el cuestionario (Alulema-Dávila \& Tinttin-Rea, 2014; Minda-Mina, 2011; Villadurna-Ríos, 2013).

En Colombia aún no se ha realizado la adaptación de este instrumento. Existen investigaciones que analizaron diversas problemáticas en población adolescente, que generalmente se centraron en sus problemas de conducta (Alba, 2007; Alonso-García \& Román-Sánchez, 2005;Ayala-Velázquez, Pedroza-Cabrera, Morales-Chainé, Chaparro-Caso-López, \& Barragán-Torres, 2002; Departamento Nacional de Estadística [DANE], 2005; Flores, 1992; Gómez-Restrepo, 2005; Profamilia, 2016; Programa Rumbos, 2001; Rabazo-Méndez, 1999; Rodríguez-Mayoral, Martínez-Arias, Díaz-Aguado, \& Moretin, 2008; Suarez \& Krauskopf; 1995; Vinaccia, Quiceno, \& Moreno-San-Pedro, 2007). Aunque estas investigaciones son sumamente importantes, dejan de lado un aspecto fundamental de la adolescencia: la capacidad de adaptación del adolescente. Para poder realizar investigaciones que incluyan esta variable, es primordial contar con una medida adecuada del constructo. Es por ello que en este trabajo se propone la adaptación psicométrica de la versión española del Cuestionario de Adaptación para Adolescentes de Bell (CAAB; Bell, 1934) realizada por Cerdá (1987), para su uso con población adolescente de la ciudad de Bogotá, Colombia. La adaptación del CAAB posibilitará contar con una herramienta adecuada para caracterizar la adaptación de los adolescentes colombianos de la ciudad de Bogotá y su estudio en relación con otras variables relevantes. Esta información podrá ser utilizada por los actores implicados en los ámbitos educativos y sociales en los que los adolescentes se ven insertos -docentes, psicólogos, psicopedagogos, familiares, grupos de pares, entre otros- para idear interven- ciones, tomar decisiones y actuar utilizando información generada desde una medida de adaptación apropiada.

\section{Método \\ Participantes}

Se trabajó con una muestra no probabilística constituida por 569 adolescentes, con edades entre 12 y 18 años $(M=15.35$; $\mathrm{DE}=2.01)$. Los participantes del estudio fueron estudiantes de escuelas primarias y secundarias y universidades públicas y privadas en la ciudad de Bogotá. El $53.8 \%$ de la muestra fueron mujeres $(n=306) y$ el $46.2 \%$ hombres $(n=262)$. En cuanto al estado civil de los participantes, el $83 \%(n=472)$ informó ser soltero, el 16.3\% $(\mathrm{n}=93)$ tenía una relación y sólo el $0.7 \%(n=4)$ vivía con su pareja en unión libre.

Con el fin de cumplir con los objetivos del estudio, se conformaron grupos de edad de acuerdo a la subetapa de desarrollo en la adolescencia (ver Tabla 1). Ciento veinte participantes se encontraban en la adolescencia temprana (21.1\%), 168 participantes se encontraban en la adolescencia nuclear $(29.5 \%)$ y 281 se encontraban en la adolescencia tardía (49.4\%).

\section{Tabla 2}

Distribución de la muestra agrupada por edad según subetapa de adolescencia.

\begin{tabular}{lcc}
\hline & Frecuencia & Porcentaje \\
\hline $\begin{array}{l}\text { Adolescencia Temprana } \\
(12-13 \text { años })\end{array}$ & 120 & 21.1 \\
$\begin{array}{l}\text { Adolescencia Nuclear } \\
(13-14 \text { años })\end{array}$ & 168 & 29.5 \\
$\begin{array}{l}\text { Adolescencia Tardía } \\
(16-18 \text { años })\end{array}$ & 281 & 49.4 \\
Total & 569 & 100 \\
\hline
\end{tabular}


En cuanto al nivel socioeconómico, en Colombia se utiliza una clasificación de acuerdo a niveles de estratificación. Los estratos socioeconómicos en los que se pueden clasificar las viviendas y/o predios son seis, a saber: 1 = bajo-bajo, 2 = bajo, $3=$ medio-bajo, $4=$ medio, $5=$ medio-alto y $6=$ alto. Los estratos 1,2 y 3 corresponden a los niveles bajos, a los que pertenecen las personas con menores recursos, quienes son beneficiarios de subsidios en los servicios públicos domiciliarios. Por su parte, los estratos 5 y 6 corresponden a estratos altos a los que pertenecen los ciudadanos con mayores recursos económicos, quienes a la vez deben pagar contribuciones sobre el valor de los servicios públicos domiciliarios. El estrato 4 no es beneficiario de subsidios ni debe pagar sobrecostos, paga exactamente el valor que las empresas definan como costo de prestación del servicio. La clasificación en estratos es una aproximación a la diferencia socioeconómica jerarquizada, es decir, de pobreza a riqueza y viceversa. Esta clasificación se basa en la Ley 142 de 1994 (régimen de servicios públicos domiciliarios) en la que se dictamina que las características físicas se utilizan como base para asignar los estratos a los inmuebles residenciales de la población colombiana. Se deben estratificar los inmuebles residenciales y no los hogares (artículo 101.2, Ley 142; DANE, 2005). De acuerdo con esta clasificación, la mayoría de la muestra estudiada se encontraba en el estrato $3(45.3 \% ; n=258)$, seguido por el estrato $2(27.4 \% ; n=156)$, el estrato $4(20,4 \%$; $\mathrm{n}=116)$, el estrato $1(4.2 \% ; \mathrm{n}=24)$, el estrato 5 $(2.5 \% ; n=14)$ y en menor porcentaje el estrato 6 $(0.2 \% ; n=1)$.

\section{Materiales}

Encuesta sociodemográfica. La misma fue diseñada ad hoc para esta investigación. Mediante ella se indagó acerca de la edad, sexo, estado civil, nivel socioeconómico y grado académico de los adolescentes.

\section{Cuestionario de Adaptación para Adolescentes}

de Bell. Se utilizó la adaptación española para elaborar una versión colombiana (Cerdá, 1987). Este test evalúa el nivel de adaptación de adolescentes de entre los 12 y 18 años de edad a través de 140 ítems agrupados en cuatro dimensiones de adaptación: familiar, de la salud, social y emocional. Para dar respuesta al cuestionario los participantes respondieron a los ítems, que constan de afirmaciones según la correspondencia o no de cada una con su forma de ser y de pensar o con sus percepciones e intereses. Las opciones de respuesta del cuestionario son $S I, N O$ y ?, este último utilizado en caso de no encontrarse seguro con su respuesta. Previo a la calificación del test deben invertirse algunos ítems cuya formulación va en sentido opuesto. La suma de las respuestas clave permite obtener el nivel de adaptación para cada una de las áreas de adaptación. A mayor puntaje en la puntuación bruta de cada escala, menor grado de adaptación.

\section{Procedimiento}

La versión preliminar del CAAB fue sometida a juicio experto, estudio piloto y administrada a una muestra descriptiva con el fin de analizar la redacción de cada uno de los ítems en el contexto colombiano y el poder discriminativo de los ítems e identificar la estructura factorial. La administración del cuestionario se realizó de manera colectiva. Se procuró la aplicación del instrumento en condiciones de adecuada iluminación, ventilación y bajos niveles de ruido para que los participantes respondieran motivados $\mathrm{y}$, por consiguiente, de forma honesta. Se solicitó un consentimiento informado, asegurando la confidencialidad de los 
datos y la posibilidad de una devolución individual de los resultados obtenidos en caso de ser solicitada. Asimismo, se informó la posibilidad de no realizar el cuestionario o dejar de responderlo en el momento en que los participantes lo consideraran pertinente.

\section{Análisis de datos}

Para obtener evidencias de validez de constructo se realizó un análisis de componentes principales. Se verificó la adecuación de la muestra mediante el estadístico Kaiser-Meyer-Olkin (KMO) y el test de esfericidad de Bartlett. Luego se analizó confiabilidad mediante el coeficiente alfa de Cronbach para cada una de las escalas. Posteriormente, se realizaron análisis de diferencias entre grupos para observar las diferencias entre las variables de sexo, y edad, para lo cual se analizaron los estadísticos descriptivos ( $t$ de Student ) y se llevó a cabo un ANOVA.

Finalmente se realizó la construcción de normas agrupando los baremos según las etapas de la adolescencia, para lo cual se emplearon los puntajes percentiles y los puntajes $\mathrm{Z}$.

\section{Resultados}

Juicio experto

El juicio experto fue realizado por cinco jueces quienes evaluaron la consigna, el formato de respuesta y el contenido de los ítems. Como resultado se modificó parte de la consigna para mayor comprensión de los participantes. En cuanto al formato, se determinó que para la aplicación del instrumento las opciones de respuesta se ubicarían al final de cada uno de los ítems con el fin de facilitar la selección de las opciones de respuesta una vez terminada la lectura del ítem, ya que de otra forma podrían funcionar como un distractor en la lectura. Se realizaron modificaciones en la redacción de los ítems en relación con la versión española, adecuándose las oraciones a los modismos colombianos y al vocabulario adolescente.

Con el fin de calcular los coeficientes Aiken, se solicitó a los jueces indicar la pertenencia de cada ítem respecto a cada subdimensión adaptativa para así valorar el grado de acuerdo. Los resultados arrojaron en general valores adecuados, excepto para el ítem $42(V=.0)$, el ítem $106(V=$ $.0)$ y el ítem $120(V=.4)$, que presentaron valores Aiken menores que .60, siendo retirados de los análisis posteriores teniendo en cuenta la no pertenencia de los ítems con cada una de las escalas.

\section{Estudio Piloto}

Con la versión post-juicio experto del instrumento se llevó a cabo un estudio piloto con 17 adolescentes quienes completaron y evaluaron la estructura del test en cuanto a la consigna, el formato de respuesta y el contenido de los ítems. Diversas observaciones promovieron modificaciones en el instrumento respecto a la descripción de la consigna en donde se aclaró a qué hacían referencia las respuestas $S I, N O$ y ? y la redacción de algunos de los ítems los cuales se adaptaron a la edad de los participantes y el contexto colombiano. No surgieron modificaciones en el formato del test.

\section{Análisis de ítems: Discriminación y homogeneidad}

Para evaluar la capacidad discriminativa de los ítems para cada una de las dimensiones que componen el instrumento como primer paso se suprimieron aquellos ítems en los cuales la frecuencia fuera mayor que $70 \%$ en una de las tres 
opciones de respuesta (Rodríguez-Sabiote, Gallardo-Vigil, Pozo-Lorente, \& Gutiérrez-Pérez, 2006). Se examinó la normalidad univariante mediante los estadísticos de contraste $(\mathrm{zG})$ para los valores de asimetría y curtosis con sus correspondientes errores típicos, esperando el rango de \pm 1,96 (Lévy-Mangin \& Varela-Mallou, 2006). Luego, se calcularon los índices de homogeneidad corregidos ( $\mathrm{IHc}$ ) por subescala teniendo en cuenta aquellos índices con valores inferiores a .30 (Martínez-Arias, 1995).

\section{Análisis factorial}

Los 137 ítems fueron sometidos a un análisis de componentes principales con rotación seleccionada varimax. Para comenzar se estudió el gráfico de sedimentación y los autovalores. Con el fin de intentar replicar la estructura de la prueba creada por Bell se forzó la extracción de cuatro factores. Se eliminaron elementos con cargas inferiores a .40 o cargas dobles y se logró aislar una estructura de 24 ítems que explicaba el $36.77 \%$ de la varianza $\left(\chi^{2}=1823,71 ; g l=276 ; p=.0001\right.$; $\mathrm{KMO}=.76)$. La estructura puede visualizarse en la Tabla 3. El Factor 1 corresponde con los ítems de la escala familiar, el Factor 2 corresponde con los ítems de la escala de salud, el Factor 3 corresponde con los ítems de la escala social y por último, el Factor 4 corresponde con los ítems de la escala emocional. También se calcularon los coeficientes alfa de Cronbach para valoración de consistencia interna. Tal como puede verse también en la Tabla 3 , se verificaron valores adecuados para todas las escalas a excepción de la escala de salud.

\section{Diferencias según sexo y edad}

Con el fin de conocer diferencias estadísticamente significativas en las escalas de adaptación familiar, de salud, social y emocional, a partir de la variable sexo se utilizó una prueba $t$ de Student para muestras independientes.

Los resultados muestran una diferencia estadísticamente significativa en las dimensiones de adaptación familiar, $t_{(567)}=-3.13, p=.002 \mathrm{y}$ adaptación emocional $t_{(567)}=-8.10 p<.001$. De acuerdo con lo anterior se encuentra que las mujeres presentan menores índices de adaptación familiar que los hombres, $\mathrm{M}_{\mathrm{v}}=1.99$ vs. $\mathrm{M}_{\mathrm{M}}=2.44 \mathrm{y}$ también menores índices de adaptación emocional, $\mathrm{M}_{\mathrm{v}}=1.98 v s . \mathrm{M}_{\mathrm{M}}=3.19$. Debe recordarse que en esta escala a mayor puntaje, menor adaptación. Al mismo tiempo, no se hallaron diferencias estadísticamente significativas en las subescalas de adaptación de salud y social $(p=.05)$.

A continuación, para verificar si existían diferencias estadísticamente significativas en las escalas de adaptación familiar, de salud, social y emocional según el grupo de edad, se calcularon pruebas ANOVA one-way. Se encontró una diferencia estadísticamente significativa en relación con la adaptación familiar, $\mathrm{F}_{(2,566)}=3.41, p<.001 \mathrm{y}$ en relación con la adaptación emocional, $\mathrm{F}_{(2,566)}=$ $11.08, p=.001$. En el caso de la adaptación familiar, la prueba post-hoc de Bonferroni indicó que existían diferencias significativas $(p=.05)$ entre la adolescencia nuclear $(\mathrm{M}=2.52)$ y la tardía $(\mathrm{M}=2.13)$. Para la adaptación emocional la prueba post-hoc indicó que existían diferencias estadísticamente significativas entre todos los pares de rangos de edad. Los que pertenecían a la adolescencia tardía mostraron más adaptación $(\mathrm{M}=$ 2.57) que la adolescencia nuclear $(\mathrm{M}=3.12)$. Así mismo, los adolescentes que se encontraron en la adaptación temprana, mostraron mayor adaptación $(\mathrm{M}=2.09)$ que los adolescentes en etapa nu- 
Tabla 3

Estructura Factorial del CAAB.

\begin{tabular}{|c|c|c|c|c|c|}
\hline \multirow{2}{*}{ Ítem } & & \multicolumn{4}{|c|}{ Factor } \\
\hline & & 1 & 2 & 3 & 4 \\
\hline Item 35. & ¿Lloras con facilidad? & & & & .56 \\
\hline Ítem 57. & ¿Te desanimas fácilmente? & & & & .65 \\
\hline Ítem 113. & $\begin{array}{l}\text { ¿Te encuentras a menudo como si sintieras por dentro una especie de } \\
\text { nerviosismo? }\end{array}$ & & & & .57 \\
\hline Ítem 123. & $\begin{array}{l}\text { Tras haber sufrido una situación humillante, ite quedas muy } \\
\text { preocupado(a) durante bastante tiempo? }\end{array}$ & & & & .64 \\
\hline Ítem 128. & ¿Tienes altibajos en tu humor sin una razón aparente? & & & & .63 \\
\hline Ítem 132. & ¿Te dan a menudo las ideas tantas vueltas en la cabeza que te impiden & & & & .53 \\
\hline
\end{tabular}

Ítem 132. ¿Te dan a menudo las ideas tantas vueltas en la cabeza que te impiden

Ítem 33. ¿Has sufrido alguna herida o lesión importante a consecuencia de algún accidente?

Ítem 38. ¿Has tenido alguna operación quirúrgica importante?

Ítem 50. ¿Has tenido dos o más enfermedades importantes durante los últimos diez años? (enfermedades graves o que te hayan obligado a guardar cama durante varias semanas)

Ítem 69. ¿Has necesitado que te visiten los médicos con bastante frecuencia?

Ítem 124. ¿Faltas a clase con cierta frecuencia por estar enfermo(a)?

Ítem 21. ¿Te parece que en tu casa debía deexistir un poco más de comprensión y de afecto?

Ítem 51. ¿Estás casi siempre en desacuerdo con la forma en que enfoca las cuestiones de casa alguno de tus padres?

Ítem 67. ¿Te parece que tus padres han sido o son demasiado severos contigo?

Ítem 72. ¿Alguno de tus padres tiene costumbres o modales que te desagradan?

Ítem 82. ¿Te ves obligado(a) de vez en cuando a callarte o a marcharte a otra habitación con la finalidad de que haya paz y tranquilidad en tu hogar?

Ítem 134. ¿Tienes frecuentemente la impresión de que tus padres no te comprenden bien?

Ítem 8. Cuando estás en una reunión o con un grupo de amigos, ¿te gusta ser la encargada de presentar unos a otros? (INVERSO)

Ítem 15. ¿Has tomado a veces la iniciativa para animar alguna reunión aburrida? (INVERSO)

Ítem 49. ¿Te resulta difícil entablar conversación con una persona que te acaban de presentar?

Ítem 70. ¿Te resulta muy difícil hablar en público?

Ítem $96 . \quad$ ¿Haces amigos(as) con facilidad? (INVERSO)

Ittem 127. ¿Te gusta asistir a fiestas y reuniones a las que acuden muchas personas? (INVERSO)

Ítem 130. ¿Te sientes seguro(a) de ti mismo(a) cuando tienes que hablar en clase? (INVERSO)

\begin{tabular}{lcccc}
\hline Alfa de Cronbach & .66 & .49 & .62 & .70 \\
\%Varianza Explicada & 9.36 & 7.21 & 9.25 & 10.95 \\
\%Varianza Total Explicada & \multicolumn{3}{c}{36.77} \\
\hline
\end{tabular}


clear. No se hallaron diferencias estadísticamente significativas en la adaptación de salud y la adaptación social en relación con el grupo de edad del adolescente $(p=.05)$.

Finalmente, se calcularon los percentiles para las puntuaciones directas de las dimensiones familiar, de salud, social y emocional, de acuerdo con cada una de las subetapas de desarrollo adolescente.

\section{Tabla 4}

Baremos del Cuestionario de Adaptación Adolescente en población bogotana.

\begin{tabular}{llllll}
\hline & & \multicolumn{4}{c}{ Subescalas } \\
\cline { 2 - 5 } & & $\begin{array}{c}\text { Fami- } \\
\text { liar }\end{array}$ & Salud & Social & $\begin{array}{c}\text { Emocio- } \\
\text { nal }\end{array}$ \\
\hline De 12 a & N & 120 & 120 & 120 & 120 \\
13 años & Media & 2.07 & .86 & 2.86 & 2.09 \\
& Mediana & 2.00 & 1.00 & 3.00 & 2.00 \\
& Moda & 1 & 0 & 3 & 1 \\
D.E & 1.719 & .946 & 1.726 & 1.670 \\
Mínimo & 0 & 0 & 0 & 0 \\
Máximo & 6 & 4 & 7 & 6 \\
Percentiles & & & \\
10 & 0 & & 0 & 0 \\
20 & & & 1 & \\
30 & & & & \\
40 & 1 & 0 & 2 & 1 \\
50 & & & & \\
60 & 2 & & 3 & 2 \\
70 & 3 & 1 & & 3 \\
80 & 4 & & 4 & \\
90 & $\geq 5$ & $\geq 2$ & $\geq 5$ & $\geq 4$ \\
\hline
\end{tabular}

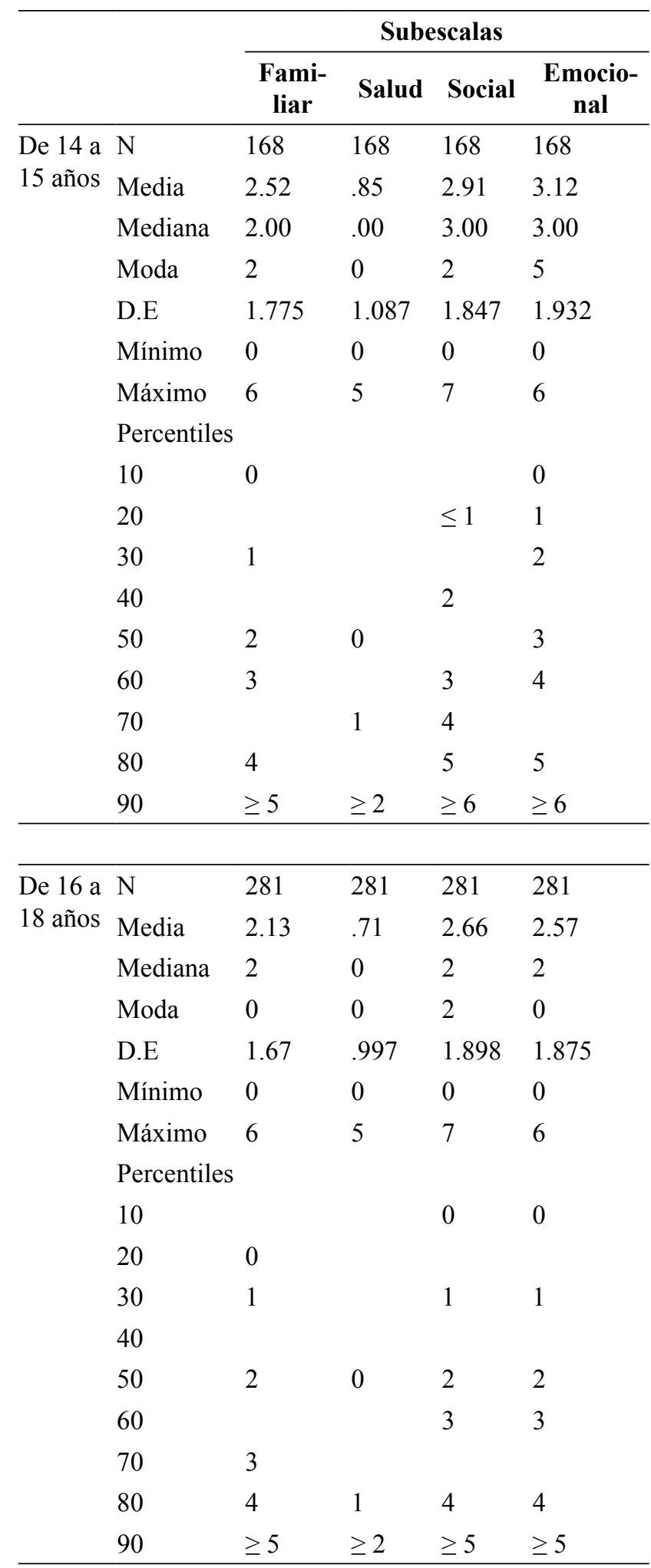




\section{Discusión}

El objetivo de este trabajo radicó en el estudio psicométrico del Cuestionario de Adaptación para Adolescentes de Bell (CAAB; Bell, 1934) en población de adolescentes bogotanos. De los análisis aquí realizados se puede concluir que la estructura tetrafactorial propuesta por Bell resultó replicable en una muestra de adolescentes bogotanos. Se realizó la modificación de la consigna y la redacción de algunos ítems para la comprensión de la población a la que va dirigida el cuestionario.

Aunque con un número menor de ítems, se replica la estructura de cuatro factores encontrada en la adaptación española realizada por Cerdá (1987) y la mexicana realizada por Del Bosque-Fuentes y Aragón-Borja (2008). Las cuatro dimensiones evalúan claramente y de manera independiente los cuatro aspectos indicados por Be11: familiar, de salud, social y emocional. Los análisis indicaron que el Cuestionario de Adaptación para Adolescentes de Bell (CAAB) posee cualidades psicométricas adecuadas -evidencias de validez de contenido, aparente y de constructo, así como alta consistencia interna- para realizar una evaluación confiable y válida del constructo adaptación en población de adolescentes bogotanos.

A pesar de que la versión utilizada es mucho más breve que la original, el contenido de cada dimensión logra abordar aquellas dimensiones postuladas originalmente por Bell (1934) y la versión española por Cerdá (1987). Esta versión nueva generada para el contexto colombiano va así en línea con los estándares vigentes internacionalmente en cuanto a la psicometría en los que se pone especial énfasis en contar con versiones más breves para acortar los tiempos de recolección de datos y favorecer la calidad de respuesta en términos de atención y colaboración del evaluado (Moore, Halle, Vandivere, \& Mariner, 2002).

Además, a partir de los resultados de las pruebas $t$, se encontraron diferencias según el sexo en las escalas emocional y familiar para la adaptación adolescente, en las cuales los varones presentaron mayores índices de adaptación que las mujeres. Estos hallazgos son semejantes a los resultados obtenidos en las investigaciones realizadas por Aragón-Borja y Bosques (2012), Funes et al. (2010) y Alonso-Fernández (2005) quienes señalan que las mujeres presentan menores niveles de adaptación en correspondencia con los cambios corporales, el afán por adaptarse al medio que propende autoevaluaciones y la relaciones familiares, las cuales cumplen un papel modelador.

En cuanto a las diferencias por edad referenciando a las subetapas de la adolescencia, se realizó una prueba ANOVA, que halló diferencias en la escala de adaptación familiar, específicamente entre las subetapas adolescencia nuclear y tardía, y en la escala de adaptación emocional en cada una de las subetapas. Los resultados muestran mayor adaptación para cada dimensión en las subetapas de adolescencia tardía y primaria frente a la adolescencia nuclear, lo que coincide con lo teóricamente expuesto por Aragón-Borja y Bosques (2012), Martínez-Antón, Buelga y Cava (2007), Cardenal-Hernáez y Fierro-Bardaji (2001), Carvajal-Corzo (1993) y Flores (1992). Esto está relacionado con los patrones representativos de las actitudes, cambios, vinculaciones y comportamientos de cada una de las subetapas de la adolescencia.

Entre las limitaciones del trabajo se encuentran el hecho de haber utilizado una muestra no probabilística para los análisis y la baja representación de los estratos 1 y 5, lo cual limita la generalización de los resultados a toda la población de adolescentes de Bogotá, Colombia. Sumado a ello, futuras investigaciones deberían valorar la estabilidad de las puntuaciones mediante un test-retest con un intervalo breve de tiempo que 
considere las diferencias en la adaptación encontradas entre las subetapas de la adolescencia. Además, sería relevante la continuidad de búsqueda de evidencias psicométricas en términos de validez para verificar, por ejemplo, la invarianza factorial en distintas muestras, así como estudios de validez convergente y discriminante.

El CAAB resulta ser así una herramienta de fácil administración y puntuación fundamentada en la concepción de la adaptación como un comportamiento universal de suma relevancia en la adolescencia (Borges del Rosal, Hernández, \& Rodríguez-Naveiras, 2011; Labajos-Alonso, 1996; Rodríguez et al., 1999). Es importante tener en cuenta que el instrumento, además de ser construido sobre este modelo, hace hincapié en la importancia que se da a la adaptación como constructo fundamentado en la autopercepción y la evaluación de sí mismo. Se tiene en cuenta un doble proceso: el ajuste a las preferencias y necesidades propias del adolescente y el ajuste de las conductas al entorno. Se debería llegar entonces a un acuerdo entre las características personales y las demandas del medio, enmarcado en el proceso ambivalente y de constante desarrollo que deviene del período adolescente (Alcalay et al., 2005; Fierro \& Cardenal 1996; García-Pérez \& Magaz-Lagos, 2001; Gómez-Bustamante \& Cogollo, 2010; Martínez-Ferrer et al., 2011).

Se espera que el instrumento aquí presentado -ver Anexo- sea de beneficio para su uso en ámbitos de aplicación y de investigación. Como se ha señalado, el grado de adaptación de los adolescentes no sólo resulta una variable central en este período del ciclo vital, sino que investigaciones precedentes lo han vinculado directamente al desenlace exitoso o fracasado en la transición por este período. El CAAB podría ser utilizado para conocer en qué áreas debe profundizarse la atención a los adolescentes, lo que podría derivar en el diseño de programas de intervención, ya sea en instituciones educativas o en ámbitos de atención de la salud, con el fin de fomentar las herramientas necesarias en el adolescente para que logre adaptarse a todos los desafíos inherentes a este período de su vida.

\section{Referencias}

Alcalay, L., Milicic, N., \& Torretti, A. (2005). Alianza efectiva familia-escuela: Un programa audiovisual para padres. Psykhe, 14(2), 149-161. doi: 10.4067/s071822282005000200012

Alba, L. H. (2007). Factores de riesgo para iniciar el consumo de tabaco. Revista Colombiana de Cancerología, 4(11), 250-257. Recuperado de http://www.cancer. gov.co/documentos/revistas/2007/pub4/5.\%20Art\%C3\%ADculo\%20de\%20revisi\%C3\%B3n.pdf

Allport, G. W., \& Allport, F. H. (1939). The A-S Reaction Study: A scale for measuring ascendance-submission in personality; manual of directions, scoring values, and norms. Boston: Mifflin.

Alonso-Fernández, M. (2005). Relaciones familiares y ajuste en la adolescencia (Tesis de doctorado). Universidad de Valladolid, España. Recuperado de https:// www.uv.es/lisis/otras-publica/tesis manuela.pdf

Alonso-García, J., \& Román-Sánchez, J. M. (2005). Prácticas educativas familiares y autoestima. Psicothema, 17(1), 76-82. Recuperado de http://www.psicothema. es

Alulema-Dávila, L., \& Tinttin-Rea, S. (2014). Adaptación, rasgos de personalidad y factores sociodemográficos comunes en los adolescentes pertenecientes a grupos étnicos católicos salesianos. (Tesis de grado). Recuperado de dspace.uazuay.edu.ec/bitstream/datos/3656/1/10333.pdf

Aragón-Borja, L. E., \& Bosques, E. (2012). Adaptación familiar, escolar y personal de a11dolescentes en la ciudad de México. Enseñanza e Investigación en Psicología, 17(2), 263-282. Recuperado de http://www. redalyc. org/articulo.oa?id=29224159002 
Atienza-González, F. L., Moreno-Sigüenza, Y., \& Balaguer-Solá, I. (2000). Análisis de la dimensionalidad de la Escala de Autoestima de Rosenberg en una muestra de adolescentes valencianos. Revista de Psicología Universitas Tarraconensis, 22(2), 29-42.

Ayala-Velázquez, H., Pedroza-Cabrera, F., Morales-Chainé, S., Chaparro Caso-López, A., \& Barragán-Torres, N. (2002). Factores de riesgo, factores protectores y generalización del comportamiento agresivo en una muestra de niños en edad escolar. Salud Mental, 25(3), 27-40. Recuperado de http://www.redalyc.org/ pdf/582/58232504.pdf

Baessler, J., \& Schwarzer, R. (1996). Evaluación de la autoeficacia: Adaptación española de la Escala de Autoeficacia General. Ansiedad y Estrés, 2(1), 1-8.

Bell, H. M. (1934). The Adjustment Inventory. California: Stanford University Press.

Bernreuter, R. (1935). Manual for the Personality Inventory. California: Stanford University Press.

Bharvad, M. B. (2015). A study of emotional intelligence and adjustment among school students. The International Journal of Indian Psychology, 2(2), 23-31. Recuperado de http://oaji.net/articles/2015/1170-1427635156. pdf

Borges del Rosal, A., Hernández, C., \& Rodríguez-Naveiras, E. (2011). Evidencias contra el mito de la inadaptación de las personas con altas capacidades intelectuales. Psicothema, 23(3), 362-367. Recuperado de http://www.psicothema.com/

Caballo, V. E., \& Ortega, A. R. (1989). La escala multidimensional de Expresión Social: Algunas propiedades psicométricas. Revista de Psicología General y Aplicada, 42(2), 215-221. Recuperado de https://dialnet. unirioja.es/servlet/articulo? codigo $=2359241$

Cardenal-Hernáez, V., \& Fierro-Bardaji, A. (2001). Sexo $\mathrm{y}$ edad en estilos de personalidad, bienestar social y adaptación social. Psicothema, 13(1), 118-126. Recuperado de http://www.psicothema.com/

Carvajal-Corzo, G. (1993). Adolecer: La aventura de una metamorfosis. Una visión psicoanalítica de la adolescencia. Bogotá: Printing Service Network.
Cerdá, E. (1987). Cuestionario de Adaptación para Adolescentes. Manual. Barcelona, España: Herder.

Coleman, J., \& Hendry, L. (1999). The nature of adolescence ( $3^{\mathrm{a}}$ ed.). Barcelona, España: Routledge.

Del Bosque-Fuentes, A. E., \& Aragón-Borja, L. E. (2008). Nivel de adaptación en adolescentes mexicanos. Interamerican Journal of Psychology, 42(2), 287-297. Recuperado de http://pepsic.bvsalud.org/pdf/rip/ v42n2/v42n2a10.pdf

Departamento Administrativo Nacional de Estadística (DANE). (2005). Análisis de la estructura y composición de las principales variables demográficas y socioeconómicas del Censo 2005. Recuperado de https:// www.dane.gov.co/files/censos/Grupo_mixto22\%20 PUBL.pdf

Diener, E., Emmons, R. A., Larsen, R. J., \& Griffin, S. (1985). The Satisfaction With Life Scale. Journal of Personality Assessment, 49(1), 71-75. doi: 10.1207/ s15327752jpa4901_13

Fernández-Ballesteros, R., \& Sierra, B. (1982). Estudio factorial sobre la percepción del ambiente escolar. En R. Fernández-Ballesteros (Ed.), Evaluación de Contextos (pp. 9-49). Murcia: Servicio de Publicaciones de la Universidad de Murcia.

Fierro, A. (1990). Autoestima en adolescentes. Estudios sobre su estabilidad y sus determinantes. Estudios de Psicología, 12(45), 85-107. doi: 10.1080/02109395.1991.10821156

Fierro, A. (1997). Estrés, afrontamiento y adaptación. En M. I. Hombrados (Ed.), Estrés y salud (pp. 11-37). Valencia, España: Promolibro.

Fierro, A., \& Cardenal, V. (1996). Dimensiones de personalidad y satisfacción personal. Revista de Psicología General y Aplicada. 49(1), 65-82. Recuperado de https://dialnet.unirioja.es/servlet/articulo?codi$\mathrm{go}=2358113$

Flores, A. (1992). Educación sexual. Uruguay: Dismar.

Funes, M. J., Lupiáñez, J., \& Humphreys, G. (2010). Analizyng the generality of conflict adaptation effects. Journal of Experimental Psycghology, 36(1), 147161. doi: 10.1037/a0017598 
García-Pérez, E. M., \& Magaz-Lagos, A. (2001). Escala Magallanes de Adaptación. Madrid, España: Albor COHS.

Gómez-Restrepo, C. (2005). Psiquiatria y salud mental de niños y adolescentes: Una necesidad. Revista Colombiana de Psiquiatría, 34(3), 339- 345. Recuperado de http://www.scielo.org.co/scielo. php?pid=S0034-74502005000300001\&script $=$ sci arttext\&tlng=pt

Gómez-Bustamante, E. M., \& Cogollo, Z. (2010). Factores predictores relacionados con el bienestar general en adolescentes estudiantes de Cartagena, Colombia. Revista de Salud Pública, 12(1), 61-70. doi: 10.1590/ s0124-00642010000100006

González, A. L., De los Ríos, D. P., \& Viveros, E. F. (2015). Crisis familiares y rendimiento académico en niños de 8 a 10 años. Una aproximación desde la dinámica interna familiar. Infancias Imágenes, 14(2), 25-36. doi:10.14483/udistrital.jour.infimg.2015.2.a02

Gutiérrez, G. (2000). Adaptación, validación y estandarización de la Escala Multidimensional de Expresión Social-Parte Motora y Parte Cognitiva en estudiantes de la Universidad Nacional de Colombia (Tesis de grado). Bogotá, Colombia: Universidad Nacional de Colombia.

Holahan, C. J., Moos, R. H., \& Schaefer, J. A. (1996). Coping, stress resistance, and growth: Conceptualizing adaptive functioning. En M. Zeidner \& N. S. Endler (Eds.), Handbook of coping: Theory, Research and Application (pp. 24-42). New York: John Wiley \& Sons.

Huebner, E. S. (1991). Initial development of the Student's Life Satisfaction Scale. School Psychology International, 12(3), 231-240. doi: 10.1177/0143034391123010

Jiménez, M. I., \& López-Zafra, E. (2011). Actitudes sociales y adaptación social en adolescentes españoles: El papel de la inteligencia emocional percibida. Revista de Psicología Social, 26(1), 105-117. doi: 10.1174/021347411794078417
Kostanski, M., \& Gullone, E. (1998). Adolescent body image dissatisfaction: Relationship with self-esteem, anxiety, and depression controlling for body mass. Journal of Child Psychology and Psychiatry, 39(2), 255-262. doi: 10.1017/s0021963097001807

Kovacs, M. (2004). Inventario de Depresión Infantil (CDI). Madrid, España: TEA Ediciones.

Kulshrestha, S. P. (1979). Educational Psychology. Meerut, India: Loyal Book Depot.

Labajos-Alonso, J. (1996). Identidad del adolescente. En Á. Aguirre (Ed.), Psicología de la Adolescencia (pp. 173-194). Bogotá, Colombia: Alfaomega.

Ley 142. (1994). Ley Sobre Servicios Públicos. Recuperado de https://www.minminas.gov.co/documents/10180/670382/LEY142DE1994.pdf/ 68f0c21d-fd78-4242-b812-a6ce94730bf1

Lévy-Mangin, J. P., \& Varela-Mallou, J. (2006). Modelización con estructuras de covarianzas en ciencias sociales.Coruña, España: Netbiblio.

Lynch, D., Norem, K., \& Gergen, R. (1981). Self-Concept. Advances in theory and research (pp. 119-13). Cambrige: Ballinger Publish.

Macías, V. T. (2000). Ser adolescente. México: Trillas.

Martínez-Arias, M. R. (1995). Psicometría: Teoría de los test psicológicos y educativos. Madrid, España: Síntesis.

Martínez-Antón, M., Buelga, S., \& Cava, M. J. (2007). La satisfacción con la vida en la adolescencia y su relación con la autoestima y el ajuste escolar. Anuario de Psicología, 38(2), 293-303. Recuperado de http:// www.redalyc.org/articulo.oa?id=97017404013

Martínez-Ferrer, B., Amador-Muñoz, L.V., Moreno-Ruiz, D., \& Musitu-Ochoa, G. (2011). Implicación y participación comunitaria y ajuste psicosocial en adolescentes. Psicología y Salud, 21(2), 205-214. Recuperado de https://www.uv.es/lisis/belen/12-psicologiasalud.pdf

Minda-Mina, J. J. (2011). Efectos emocionales presentes en adolescentes de doce a quince años de edad, procedentes de hogares disfuncionales en los que cuentan con solo la madre o el padre y su influencia en el 
comportamiento y adaptación, en el Instituto Tecnológico Superior Los Shyris, Año lectivo 2010 - 2011. (Tesis de grado). Recuperado de www.dspace.uce. edu.ec/bitstream/25000/1399/1/T-UCE-0007-6.pdf

Moos, R. H. (1974). The Social Climate Scales: An overview. Palo Alto, California: Consulting Psychologists Press.

Moraleda, M., González-Galán, A., \& García-Gallo, J. (1998). AECS. Actitudes y estrategias cognitivas sociales. Madrid, España: TEA.

Moore, K. A., Halle, T. G., Vandivere, S., \& Mariner, C. L. (2002). Scaling back survey scales. How short is too short? Sociological Methods \& Research, 30(4), 530567. doi: 10.1177/0049124102030004003

Musitu, G., \& García, F. (2001). ESPA29: Escala de Estilos de Socialización Parental en la Adolescencia. Madrid, España: TEA

Ochoa, M. A. (2012). Adaptación de adolescentes en riesgo de delincuencia. (Tesis de grado). Universidad Rafael Landivar. Guatemala.

Oliva-Delgado, A., \& Parra-Jiménez, A. (2004). Contexto familiar y desarrollo psicológico durante la adolescencia. En E. Arranz (Ed.), Familia y desarrollo psicológico (pp. 96-123). Madrid, España: Pearson Prentice-Hall.

Orte-Socías, C., \& March-Cerdá,M. (1996). Pedagogía de la Inadaptación Social: Educación Social. Valencia, España: Nau Libres.

Palmero, F., \& Fernández E. (1998). Emociones y adaptación. Barcelona, España: Ariel.

Petersen, A. C., \& Crockett, L. (1985). Pubertal timing and grade efects on adjustment. Journal of Youth and Adolescence, 14(3), 191-206. doi: 10.1007/ bf02090318

Profamilia (2016). Informe Anual de Actividades. Recuperado de http://www.profamilia.org.co/docs/INFORME\%20PROFAMILIA\%202016\%20VERSION\%20MARZO\%2016.pdf

Programa Rumbos (2001). Encuesta nacional sobre consumo de sustancias psicoactivas en jóvenes de 10-24 años. Recuperado de https://www.unodc.org/docu-
ments/colombia/Documentostecnicos/Estudio_Consumo_Escolares.pdf

Rabazo-Méndez, M. (1999). Interacción Familiar, Competencia socio-escolar y comportamiento disocial en adolescentes (Tesis Doctoral). Universidad de Extremadura, Facultad de Educación. Departamento de Psicología y Sociología de la Educación. Recuperado de https://dialnet.unirioja.es/descarga/tesis/337.pdf

Rodríguez-Sabiote, C., Gallardo-Vigil, M. A., Pozo-Lorente, T., \& Gutiérrez-Pérez, J. (2006). Iniciación al análisis de datos cuantitativos en educación. Análisis descriptivo básico: teoría y práctica mediante SPSS. Recuperado de http://www.ugr.es/ erivera/PaginaDocencia/Posgrado/Documentos/ClementeCuadernoDescriptiva.pdf

Rodríguez, M. C., Jiménez, M. A., Fernández, E., \& Godoy, C. (1999). Evaluación de psicopatología en la infancia y adolescencia a través de la percepción de los padres. Revista Iberoamericana de Diagnóstico y Evaluación Psicológica, 8(2), 51- 65. Recuperado de http://www.aidep.org/03_ridep/R08/R083.pdf

Rodríguez-Mayoral, J. M., Martínez-Arias, R., Díaz-Aguado, M. J., \& Morentín, R. (2008). Comportamiento Violento en Adolescentes: Su relación con las estrategias cognitivas y el rendimiento académico. Psicología Educativa, 14(1), 63-81.

Ros-Rahola, R., Morandi-Garde, T., Cozzetti-Sueldo, E., Lewintal-Blaustein, C., Cornella i Canals, J., \& Surís- Granell, J.C., (2001). La adolescencia: Consideraciones biológicas, psicológicas y sociales. En C. Buil-Rada, I. Lete-Lasa, R. Ros-Rahola \& J. L. De Pablo-Lozano (Eds.), Manual de Salud Reproductiva en la Adolescencia (pp. 27-84). Wyeth-Lederle: España.

Rosenberg, M. (1966). Society and the adolescent self-image. Princeton: University Press.

Siverio-Eusebio, M. A., \& García-Hernández, M. D. (2007). Self-perception of adjustment and sadness in adolescence. Anales de Psicología, 23(1), 41-48. Recuperado de http://www.um.es/analesps/v24/v24_1i.htm 
Suárez, E., \& Krauskopf, D. (1995). El enfoque de riesgo y su aplicación a las conductas del adolescente. Una perspectiva psicosocial. En M. Maddaleno, M. Munist, C. Serrano, T. Silver, E. Suárez \& J. Yunes (Eds.), La salud del Adolescente y el Joven. Washington, O.P.S. Publicación científica ${ }^{\circ}$ 552, pp. 183193.

Thurstone, L.L. (1930). Instructions for using the Personality Schedule. Chicago, Illinois: Univ. of Chicago Press.

Valverde, J. (1988). El proceso de inadaptación social. Madrid, España: Popular.

Villarduna-Ríos, M. M. (2013). Adaptación del clima social familiar en padres de familia de instituciones educativas nacionales del distrito de San Martín. Minds, 1(1), 45-65. http://ojs.ucvlima.edu.pe/index.php/ minds/article/view/15

Vinaccia, S., Quiceno, J. M., \& Moreno-San-Pedro, E. (2007). Resiliencia en Adolescentes. Revista Colombiana de Psicología, 16, 139-146. Recuperado de http://www.redalyc.org/pdf/804/80401610.pdf 


\section{Anexo 1}

\section{Cuestionario de Adaptación para adolescentes BELL - CAAB}

Te presentamos una serie preguntas que tienen como fin conocer tu percepción acerca de ti mismo y del ambiente en general. Te pedimos que respondas las preguntas con toda sinceridad, teniendo en cuenta que no hay respuestas buenas ni malas, por lo que debes marcar tu respuesta según tu realidad y no la que quisieras que fuera.
Marca en la casilla correspondiente a "SI", "NO" o "?"”, según sea tu respuesta. Debes responder siempre que te sea posible "SI" o "NO" y ÚNICAMENTE marcarás el signo de interrogación "?.” en aquellos casos en que estés completamente seguro de no poder responder ni "SI" ni “NO”. Si no vives en casa de tus padres, las preguntas que hagan referencia a tu hogar contéstalas con relación a las personas con las que convivas.

\begin{tabular}{|c|c|c|c|c|}
\hline Ítem & Afirmación & SI & NO & ? \\
\hline 1 & $\begin{array}{l}\text { Cuándo estás en una reunión o con un grupo de amigos, } \\
\text { ¿te gusta ser el que presenta unos a otros? }\end{array}$ & & & \\
\hline 2 & ¿A veces has tomado la iniciativa para animar alguna reunión aburrida? & & & \\
\hline 3 & ¿Crees que en tu casa debería existir un poco más de comprensión y de afecto? & & & \\
\hline 4 & ¿Sufres con frecuencia heridas o lesiones importantes en tu cuerpo? & & & \\
\hline 5 & ¿Lloras con facilidad? & & & \\
\hline 6 & ¿Has tenido alguna operación quirúrgica importante? & & & \\
\hline 7 & ¿Te resulta difícil entablar conversación con una persona que acabas de conocer? & & & \\
\hline 8 & $\begin{array}{l}\text { ¿Has tenido dos o más enfermedades importantes durante los últimos } 10 \text { años? } \\
\text { (enfermedades graves o que te hayan obligado a guardar cama durante varias semanas) }\end{array}$ & & & \\
\hline 9 & ¿Estás casi siempre en desacuerdo con la forma en que tus padres tratan los asuntos de casa? & & & \\
\hline 10 & ¿Te desanimas fácilmente? & & & \\
\hline 11 & ¿Te parece que tus padres han sido o son demasiado severos contigo? & & & \\
\hline 12 & ¿Has necesitado visitar al médico con bastante frecuencia? & & & \\
\hline 13 & ¿Te resulta muy difícil hablar en público? & & & \\
\hline 14 & ¿Alguno de tus padres tiene costumbres o modales que te desagradan? & & & \\
\hline 15 & $\begin{array}{l}\text { ¿De vez en cuando te ves obligado a callarte o a marcharte a otra habitación } \\
\text { con la finalidad de que haya paz y tranquilidad en tu hogar? }\end{array}$ & & & \\
\hline 16 & ¿Haces amigos con facilidad? & & & \\
\hline 17 & ¿Sientes a menudo nerviosismo? & & & \\
\hline 18 & $\begin{array}{l}\text { Luego de haber sufrido una situación humillante, ¿te quedas } \\
\text { muy preocupado durante bastante tiempo? }\end{array}$ & & & \\
\hline 19 & ¿Faltas a clase con cierta frecuencia por estar enfermo? & & & \\
\hline 20 & ¿Te gusta asistir a fiestas y reuniones a las que acuden muchas personas? & & & \\
\hline 21 & ¿Tienes altibajos de humor sin alguna razón aparente? & & & \\
\hline 22 & ¿Te sientes seguro de ti mismo cuando debes hablar en clase? & & & \\
\hline 23 & $\begin{array}{l}\text { A la hora de dormir, ¿Tienes a menudo muchas ideas en tu cabeza a las } \\
\text { que le das tantas vueltas que te impiden dormir normalmente? }\end{array}$ & & & \\
\hline 24 & ¿Frecuentemente tienes la impresión de que tus padres no te comprenden bien? & & & \\
\hline
\end{tabular}




\section{Clave de corrección}

Sumar los ítems de cada dimensión de acuerdo a la siguiente codificación: $\mathrm{NO}=0, ?=1, \mathrm{SI}=1$. Previo a ello deben invertirse las puntuaciones de los ítems que tienen la letra $\mathrm{R}(\mathrm{NO}=1, ?=1$, SI $=0)$.

\begin{tabular}{ll}
\hline \multicolumn{1}{c}{ Dimensión del CAAB } & \multicolumn{1}{c}{ Número de Ítem } \\
\hline Factor 1. Familiar & $3,9,11,14,15,24$ \\
Factor 2. Salud & $4,6,8,12,19$ \\
Factor 3. Social & $1 \mathrm{R}, 2 \mathrm{R}, 7,13,16 \mathrm{R}, 20 \mathrm{R}, 22 \mathrm{R}$ \\
Factor 4. Emocional & $5,10,17,18,21,23$ \\
\hline
\end{tabular}

